Fernanda Cristina Barbosa Pereira Queiroz ${ }^{1}$

Hélio Roberto Hékis ${ }^{2}$

Jamerson Viegas Queiroz ${ }^{3}$

Lucas Ambrósio B. de Oliveira ${ }^{4}$

Natália Veloso Caldas de Vasconcelos ${ }^{5}$

\title{
Contribuição dos Sistemas Integrados de Gestão para as Práticas de Ensino e Aprendizagem
}

\section{Resumo}

Este artigo tem como objetivo evidenciar a importância que um sistema de gestão tem para uma IES, e como este sistema, pode colaborar com as práticas de ensino e aprendizagem, e isto será feito através de um estudo de caso na Universidade Federal do Rio Grande do Norte. Como justificativa para o estudo temos a universidade como um ambiente nos quais muitas pessoas tem pouco tempo para desenvolver as atividades, o uso de ferramentas evoluídas tecnologicamente, neste caso o sistema de gestão, facilitaria bastante o cotidiano das pessoas. A problemática de pesquisa gira em torno do seguinte questionamento: como um sistema de informação pode contribuir para as práticas de ensino. A metodologia empregada consistiu em duas etapas, revisão bibliográfica e estudo de caso. Na Universidade Federal do Rio
Grande do Norte sendo que o objeto de estudo foi o Sistema Integrado de Gestão de Atividades Acadêmicas, o (SIGAA). Os resultados encontrados evidenciaram a importância de um sistema de gestão para a prática do ensino e aprendizagem, e isso foi feito através da descrição do sistema de informação, mostrando a utilidade de seus módulos. A conclusão que pode ser feita é que o sistema de informação integrado a gestão universitária, envolve processos mutuamente dependentes de credibilidade, agilidade e recuperação rápida das informações, colaborando também para o relacionamento professor-aluno, tornado os processos mais eficientes.

Palavras-chave: Gestão Universitária; Sistemas de gestão; Ensino.

\footnotetext{
${ }^{1}$ Fernanda Cristina Barbosa Pereira Queiroz - Doutora e Mestre em Engenharia de Produção pela Universidade Federal de Santa Catarina (2003). Email: fernandacbpereira@yahoo.com.br

2 Hélio Roberto Hékis - Doutor em Engenharia de Produção e Serviços. Mestre em Administração - Gestão Estratégica das Organizações. Contador e Auditor CRC-SC 14.844; Professor do Programa de Pós-graduação em Engenharia de Produção da - UFRN; Pesquisador do Laboratório de Inovação Tecnológica em Saúde (LAIS-UFRN); Avaliador Institucional e de Cursos do INEP/MEC; Cel: +55(84) 8116-6096; Sites: http://lattes.cnpq.br/9599726799047515 http://www.docente.ufrn.br/hrhekis

3 Jamerson Viegas Queiroz - Doutor em Engenharia de Produção e mestre em Economia pela Universidade Federal de Santa Catarina. Email: jvqjamerson@yahoo.com.br

${ }^{4}$ Lucas Ambrósio B. de Oliveira - Mestrando em Engenharia de Produção na Universidade Federal do Rio Grande do Norte. Email: lucasambro@gmail.com

${ }^{5}$ Natália Veloso Caldas de Vasconcelos - Mestranda em Engenharia de Produção na Universidade Federal do Rio Grande do Norte. Email: nataliaveloso@hotmail.com
} 
CONTRIBUTION OF INTEGRATED SYSTEMS MANAGEMENT PRACTICES FOR TEACHING AND LEARNING

\section{Abstract}

This article aims to highlight the importance of a management system for an HEI has, and how this system can work with the practices of teaching and learning, and this will be done through a case study at Federal University of Rio Grande do North. As justification for the study have the university as an environment in which many people have little time to develop the activities, the use of technologically advanced tools, case management system, greatly easing the daily lives of people. The issue of research revolves around the question: as an information system can contribute to teaching practices. The methodology used consisted of two phases, literature review and case study. At the Federal University of Rio Grande do Norte and the object of which was to study the Integrated Management of Academic Activities, the (follow). The results showed the importance of a management system for the practice of teaching and learning, and this was done through the description of the information system, showing the usefulness of its modules. The conclusion that can be made is that the integrated information system to the university administration, involves mutually dependent processes of credibility, agility and quick retrieval of information, also contributing to the teacher-student relationship, making processes more efficient.

Keywords: University Management, Systems Management, Education.

\section{Introdução}

Tem sido notável o avanço da internet para áreas diferenciadas do conhecimento a partir de 1995. A ampliação da utilização da internet do âmbito acadêmico para a sociedade em geral, com sua posterior entrada na área governamental, figurou o início do uso de novas tecnologias da informação na prestação de serviços, como também de oferta de informações para cidadãos, fornecedores e servidores.

Segundo Nascimento e Araújo (2006), o ensino superior no país tem passado por diversas dificuldades, sejam elas financeiras, de planejamento, ou até de redefinição nas metodologias de ensino, algumas instituições encontram, por iniciativas próprias, formas para dinamizar o ensino, auxiliando diretamente na melhoria da qualidade na formação dos acadêmicos.

A busca das instituições de ensino superior, por melhores formas de gerenciamento de informações, pode ser justificada pelo fato entender como a construção de percepção de qualidade de serviço é particularmente fundamental na gestão de serviços educacionais (RUBINSZTEJN e PALACIOS, 2010). Ainda conforme esses últimos autores, essas iniciativas tornam-se possíveis através de projetos, voltados para a melhoria do ensino. Os objetivos desse tipo de projeto visam, basicamente, dinamizar as aulas e preparar o aluno para a realidade do mercado de trabalho, tornando o estudo cada vez mais prático, em vez do estilo tradicional de ensino, que ainda é muito utilizado no país. Sendo assim, tem-se uma reestruturação da metodologia do ensino na graduação.

Conforme Burgarelle e Carvalho (2006) o avanço das Tecnologias de Informação e Comunicação (TICs) impactou toda a sociedade com a perspectiva de acesso a informações para melhoria de vida e trabalho. O processo de globalização, aliada ao avanço das TICs, transformou a forma de competição no mercado, seja ela entre empresas, ou entre Instituições de Ensino Superior (IES), essas que passaram a depender da criação impulsionadora de novos conhecimentos e da inovação para otimização de processos, 
buscando a melhoria de produtos e serviços para satisfação dos clientes.

Devido à demanda por disseminação de conhecimento, as IES estão buscando auxílio junto às ferramentas tecnológicas, como exemplo os sistemas de informação acadêmica, que auxiliam e ampliam as atividades de geração, organização e transferência do conhecimento para fora do limite físico da sala de aula.

De acordo com as informações explanadas anteriormente, o pressente artigo tem como objetivo evidenciar a importância que um sistema de gestão tem para uma IES, e como este sistema, pode colaborar com as práticas de ensino e aprendizagem. Essa análise será feita através de um estudo de caso na Universidade Federal do Rio Grande do Norte. Buscou-se ainda identificar as melhorias que um sistema de gestão traz para as IES, no que diz respeito à agilidade nos processos $\mathrm{e}$ confiabilidade de dados.

Como justificativa para o estudo temos a universidade como um ambiente nos quais muitas pessoas tem pouco tempo para desenvolver as atividades, o uso de ferramentas evoluídas tecnologicamente, neste caso o sistema de gestão, facilitaria bastante o cotidiano das pessoas, facilitando o acesso à informação e contribuindo também para a comunicação efetiva de alunos e professores. Considerando o que já foi explanado, o cerne desta pesquisa gira em torno do seguinte questionamento: como um sistema de informação pode contribuir para as práticas de ensino.

A organização do presente estudo inicia-se com essa abordagem introdutória, seguida pela seção 2, fundamentos teóricos, na qual se aborda: práticas de ensino e aprendizagem, tendo a intenção de aprimorar conceitos hoje utilizados por estudiosos da área. A seção 3 ainda referente ao corpo teórico do estudo aborda conceitos de sistema de informação gerencial no escopo das práticas de ensino e aprendizagem. Posteriormente, a seção 4, metodologia, traz os métodos de pesquisas utilizados durante o atual estudo. Na seção 5, encontra-se o estudo de caso, mostrando como o sistema de informação utilizado pela UFRN colabora com as práticas de ensino. Em seguida, os resultados dessas análises podem ser observados na seção 6 e as considerações finais encontram-se na seção 7. Finalizando na seção Referências são apresentadas as bibliografias utilizadas na pesquisa.

\section{Práticas de ensino e aprendizagem}

Conforme Miranda (2010), o processo de Ensino-Aprendizagem é um dos campos mais férteis de contribuições nos últimos anos no Brasil. Nesse sentido, muitos focos de preocupações já foram levantados no interior do referido processo, bem como muitas foram as descobertas no sentido de melhorar sua qualidade.

Destarte, a preocupação sobre esse processo não se limita ao Brasil, em um estudo recente patrocinado pela Comissão Européia reconhece que, nos últimos anos, numerosas pesquisas mostraram uma preocupante queda de interesse dos jovens para o estudo científico (ROCARD et al., 2007 apud VILCHES, 2010). Ainda de acordo com esse autor, o citado processo tem sido alvo de críticas, em grande parte, devido ao seu distanciamento das questões práticas do dia-a-dia, como afirma Cole (1990). Para o referido autor, numa perspectiva sócio-histórica, a atividade prática está inserida no sistema de relações sociais e dele é dependente (tais como a aprendizagem), representando a unidade, e sem a qual as ferramentas oferecidas pela educação formal "enferrujam" e "caem em desuso" (COLE, 1990).

De acordo com Vasconcelos (2006) as IES são fontes multiplicadoras do saber e forte formadoras de opinião, e, por conseguinte, responsáveis por prepararem as pessoas para aprenderem a agir e a pensar por conta própria. As IES têm o compromisso de 
promover, constantemente, a produção de novos conhecimentos, o compartilhamento e o uso desse conhecimento.

Segundo Burgarelle e Carvalho (2006) existem várias tecnologias baseadas na Web como o e-learning e o portal corporativo que podem apoiar iniciativas de aprendizagem e gestão do conhecimento no ambiente acadêmico, pois essas ferramentas promovem acesso, compartilhamento de informações acadêmicas e conhecimentos de professores para professores, entre alunos e, professores e alunos.

Neste estudo será focado, no escopo da tecnologia de informação, o papel do sistema de informação acadêmico, dentro do processo ensino-aprendizagem, bem como as contribuições deste sistema, visando facilitar o processo de ensino e aprendizagem. $\mathrm{Na}$ seção seguinte, alguns conceitos sobre sistemas de informações.

\section{Sistema de informação gerencial}

De acordo com Schimitt (2004) grande parte das empresas está ciente da importância das informações para o seu processo de gestão e tomada de decisão, mas parece não se dar conta que o valor da informação está no seu uso e não na sua geração.

As transformações no ambiente empresarial acarretam para as organizações reações mais rápidas e mais inteligentes. A gestão da informação reforça seu papel fundamental na adequação às mudanças impostas pelo mercado (ROSINI e PALMISANO, 2003).

De acordo com Ochoa e Ochoa (2010) supõe-se que as tecnologias TIC's devem ser inseridas no mundo da educação através da sua presença nas IES. Enormes esforços têm sido feitos para integrá-los, seja através de programas governamentais ou de esforços, todas destinadas a proporcionar recursos para IES, através de recursos materiais e humanos, de forma visível, que vai resultar em melhorias no processo de ensino, educação e aprendizagem. Ainda conforme o autor, esta suposição é explicada pelas necessidades exigentes de sociedade de hoje, onde a informação torna-se um recurso fundamental para a integração cultural dos indivíduos, resultando em necessidade de melhorar as capacidades do seu acesso aos conhecimentos e conhecimentos através dessas ferramentas tecnológicas.

Buscando estreitar a relação entre sistema de informação gerencial, e as IES, Almeida (2002) diz que existem no mercado softwares disponíveis para o controle integrado na gestão de pequenas empresas, bem como para organizações de serviços, no caso as Instituições de Ensino Superior (IES), considerando desde estoques, controles financeiros, geração de faturas e pagamentos, o que facilita o trabalho de gestores destas organizações.

Segundo Tachizawa (2003) a gestão em ensino compreende um conjunto de decisões as quais propiciam um equilíbrio de interesses e forças dinâmicas dentro de um universo de pesquisa, ensino e extensão. A gestão passa a ser enfocada de forma sistêmica, ou seja, entendida como um conjunto de decisões assumidas a fim de obter um equilíbrio dinâmico entre missão, objetivos, meios e atividades acadêmicas e administrativas. Tachizawa (2003) também ressalta que o enfoque sistêmico propicia uma visão macroscópica da instituição de ensino, que é o ponto de partida para concepção do modelo de gestão que possibilitaria à IES responder eficazmente à nova realidade, de concorrência acirrada e de mudanças nas expectativas dos clientes.

\section{Metodologia}

A pesquisa realizada teve uma abordagem do tipo qualitativa. A classificação da pesquisa levando em consideração os objetivos tem caráter exploratório. Quanto ao planejamento 
da pesquisa optou-se pela pesquisa bibliográfica e pelo estudo de caso. A instituição que escolhida para o estudo de caso foi a Universidade Federal do Rio Grande do Norte, localizada em Natal, no nordeste do Brasil, abordando o Sistema Integrado de Gestão Acadêmica da IES desenvolvido pela Instituição. A metodologia da pesquisa descreve as etapas que serão utilizadas no decorrer da pesquisa que o objetivo seja cumprido, ou seja, compreender e conceituar práticas de ensino e aprendizagem e sistema informação gerencial; analisar o sistema utilizado pela UFRN, bem como seus relatórios e indicadores gerados pelo próprio sistema; apresentar de que maneira o sistema de informação contribui para a relação ensino e aprendizagem.

\subsection{Estudo de caso}

Atualmente a UFRN oferece 75 cursos de graduação presencial e 73 cursos de pósgraduação. Sua comunidade acadêmica é formada por mais de 33.000 estudantes (graduação e pós-graduação), 3.108 servidores técnico-administrativos e 1.760 docentes. A universidade está passando por um período de crescimento acelerado, e parte desse crescimento está associada ao Programa REUNI, por meio do qual, no ano de 2008, o projeto viabilizou a criação de 16 novos cursos de graduação nos campi de Natal, Caicó, Currais Novos, Santa Cruz e Macaíba (Jundiaí).

A ampliação em cursos novos e em cursos existentes gera 2.700 novas vagas até o ano de 2012. Tendo em vista esse cenário e a necessidade de sistemas integrados cada vez mais eficientes e que facilitem a tomada de decisões, a Universidade desenvolveu um Sistema Institucional Integrado de Gestão que é composto por cinco sistemas, direcionados a públicos diferentes, com utilidades diferentes e com vários níveis de acesso, a saber: SIPAC (Sistema Integrado de Patrimônio, Administração e Contratos); SIGAA (Sistema Integrado de Gestão de Atividades Acadêmicas); SIGPRH (Sistema Integrado de Gestão, Planejamento e Recursos Humanos); SIGAdmin (Sistema de Administração dos Sistemas - Técnica e Gestão); SIGED (Gerenciador Eletrônico de Documentos). Além desses sistemas, existe o iProject (Sistema Integrado de Gerência de Projetos) que foi desenvolvido com objetivo de gerenciar os processos de desenvolvimento dos demais sistemas (SINFO, 2011).

Porém o objeto deste estudo é o Sistema Integrado de Gestão de Atividades Acadêmicas (SIGAA). O SIGAA é o sistema responsável pelos procedimentos ligados à gestão acadêmica e possui trinta e um módulos que estão relacionados com a natureza da atividade que é oferecida, a saber: graduação, pós-graduação (stricto e lato sensu), ensino técnico, ensino médio e infantil, submissão e controle de projetos e bolsistas de pesquisa, submissão e controle de ações de extensão, submissão e controle dos projetos de ensino (monitoria e inovações), registro e relatórios da produção acadêmica dos docentes, atividades de ensino a distância e um ambiente virtual de aprendizado (chamado de Turma Virtual), dentre outros (SINFO, 2011).

Em face da importância desse módulo, para o desenvolvimento de atividades acadêmicas, envolvendo discentes, docentes e coordenações de curso e departamentos, justifica-se a escolha dos mesmos para análise neste artigo: pelo fato de ser um dos principais sistemas utilizados na Instituição, e o que principal no que tangem a áreas de gestão acadêmica da Universidade, de tal forma que influência diretamente na relação professoraluno, e pode influenciar na relação de aprendizagem dos estudantes. No capítulo seguinte, serão apresentados os resultados obtidos a partir da análise em questão. 


\section{Resultados}

A pesquisa pode comprovar a relevância que o Sistema Integrado de Gestão e Atividades Acadêmicas tem para os alunos, devido ao acesso às informações acadêmicas como notas, freqüência, calendário e plano de ensino. Para quantificar melhor esta informação, abaixo segue a tabela 1 , na qual apresenta a quantidade de acesso que o SIGAA tem por dia, sendo organizado de acordo com os dias da semana, vale ressaltar que não somente os alunos acessam os sistemas, assim como professores, e colaboradores de apoio, como as secretarias dos departamentos e dos centros.

\begin{tabular}{|c|l|}
\hline Média de Acesso/Dia & \multicolumn{1}{|c|}{ Dias } \\
\hline 1.318 .226 & Segunda \\
\hline 1.255 .088 & Terça \\
\hline 1.230 .030 & Quarta \\
\hline 1.153 .171 & Quinta \\
\hline 978.386 & Sexta \\
\hline 570.564 & Sábado \\
\hline 519.711 & Domingo \\
\hline
\end{tabular}

Tabela 1 - Média de acesso ao SIGAA, por dia. Fonte: SINFO (2011).

Em posse das informações sobre o acesso ao sistema, é possível identificar que o mesmo é acessado por grande parte dos alunos, professores e colaboradores da universidade, sendo isto positivo para o desenvolvimento das atividades da disciplina, visto o acesso ao sistema pelos alunos é um indicativo de interesse pelos assuntos da universidade.

$\mathrm{Na}$ figura 1, abaixo, é possível observar quais campos o SIGAA possui, e como o professor pode interagir com o aluno, buscando modernizar esta relação, contribuindo para a melhora da mesma.

$O$ aluno ao se matricular em uma disciplina, em qualquer que seja, tem um ambiente como o descrito na figura 1, onde no canto esquerdo, é possível observar campos como, plano de curso, onde o professor informa carga horária, ementa, objetivos e cronograma da disciplina, o campo, participantes, onde o aluno pode acessar quais os componentes da turma, o fórum, onde é permitida a criação de um ambiente de interação entre alunos e professor, e o campo chat, que é um bate-papo em tempo real. Outro importante recurso que o SIGAA tem é o de inserção de documentos, o professor pode disponibilizar arquivos para os alunos, independente do formato do arquivo. É válido lembrar que esses recursos são disponíveis somente aos alunos matriculados na disciplina e ao professor ministrante da mesma.

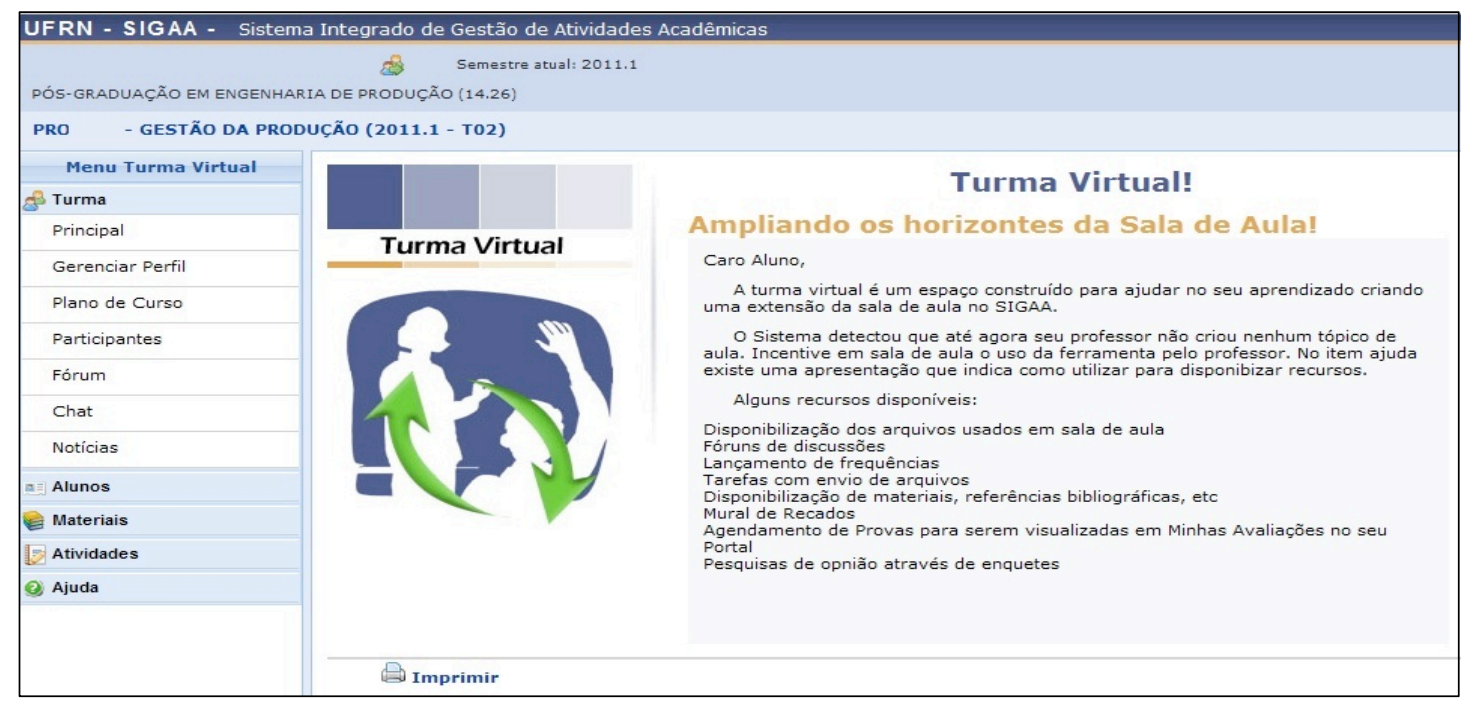

Figura 1 - MODELO SIGAA.

Fonte: Site do SIGAA (2011) 
Para quantificar o que foi dito sobre os recursos que o SIGAA oferece, a tabela 2 abaixo mostra a relação entre as disciplinas oferecidas, disciplinas em que os professores utilizam os recursos do SIGAA (em específico, a parte de inserção de arquivos) e o número de disciplinas que utilizam o fórum.

\begin{tabular}{|l|r|r|}
\hline Total de Turmas Cadastradas & \multicolumn{2}{|c|}{6.326} \\
\hline $\begin{array}{l}\text { Turmas que utilizam inserção de } \\
\text { documentos }\end{array}$ & 2.832 & $45 \%$ \\
\hline Turmas que utilizam fóruns & 949 & $15 \%$ \\
\hline
\end{tabular}

Tabela 2 - Informações sobre turmas que utilizam o SIGAA.

Fonte: SINFO (2011)

O diagnóstico feito perante o SIGAA é que o sistema contribui para a melhor interação aluno e professor, assim como, através de seus recursos, ajuda na aprendizagem do aluno ao fornecer um contato mais fácil e rápido com os professores por meio de suas ferramentas (fórum e chat), e permitem ao professor uma forma rápida e fácil de disseminar materiais e arquivos que podem ajudar aos alunos, isso tudo, através da internet: fonte de informação mais utilizada hoje no mundo todo. Para explicitar esta contribuição do sistema para a relação de professor-alunos, a tabela 3 abaixo, explana o volume de acesso ao sistema acadêmico, por professores e por aluno, por dia.

\begin{tabular}{|l|r|}
\hline Volume de Acesso de Discentes & 808.068 \\
\hline Volume de Acesso de Docentes & 80.672 \\
\hline Total de Acesso & 897.740 \\
\hline
\end{tabular}

Tabela 3 - Informações sobre discentes e docentes que utilizam o SIGAA.

Fonte: SINFO (2011)

\section{Considerações Finais}

Considerando o objetivo geral do presente estudo, ou seja, evidenciar a importância que um sistema de gestão tem para uma IES e como este sistema pode colaborar com as práticas de ensino e aprendizagem, isto foi realizado por meio de um estudo de caso na Universidade Federal do Rio Grande do Norte. Apresentaremos, a seguir, os aspectos mais marcantes nesta área de atuação.

Buscou-se identificar as melhorias que um sistema de gestão traz para as IES, no que diz respeito à agilidade nos processos e confiabilidade de dados. Assim, o estudo justificou-se pela possibilidade de podermos analisar a Universidade Federal do Rio Grande do Norte - UFRN, como um ambiente em que muitas pessoas têm pouco tempo para desenvolver as atividades, o uso de ferramentas evoluídas tecnologicamente, neste caso, o sistema de gestão facilitaria bastante o cotidiano das pessoas, facilitando o acesso à informação e contribuindo também para a comunicação efetiva de alunos e professores.

Neste contexto, o objeto de estudo foi o Sistema Integrado de Gestão de Atividades Acadêmicas (SIGAA), do qual destacamos que os resultados encontrados evidenciaram a importância de um sistema de gestão para a prática do ensino e aprendizagem, e isso foi feito através da descrição do sistema de informação, mostrando a utilidade de seus módulos.

Conclui-se dessa maneira, que o sistema de informação integrado à gestão universitária envolve processos mutuamente dependentes de credibilidade, agilidade e recuperação rápida das informações, colaborando para o relacionamento professor-aluno, tornado os processos mais eficientes e eficazes.

Por fim, conclui-se também, que o diagnóstico feito perante o SIGAA é que o sistema contribui para a melhor interação aluno e professor, assim como, através de seus recursos, ajuda na aprendizagem do aluno ao fornecer um contato mais fácil e rápido com os professores por meio de suas ferramentas (fórum e chat), e permite ao professor uma forma rápida e fácil de disseminar materiais e arquivos que podem ajudar aos alunos. 


\section{Referências Bibliográficas}

ALMEIDA, M. de S. Administração estratégica de sistemas de informações. Florianópolis: [s.n.] (apostila), 2002.

BURGARELLE, R.; CARVALHO, R.B. Avaliação do uso de sistemas de informação acadêmica por alunos de graduação em Ciência da Informação. VII ENANCIB, realizado de 19 a 22 de Novembro de 2006 - Disponível em: <http://portalppgci.marilia.unesp.br/enancib/viewabstract.php? id=224>. Acesso em: 12 mar. 2011.

COLE, M. Cognitive development and formal schooling: the evidence from cross-cultural research. In: MOLL, L.C.(ed.). Vygotsky and education. New York: Cambridge University Press, 1990. pp. 89-108.

MIRANDA, S.G. Língua e linguagem: uma reflexão acerca da dialética ensino aprendizagem. Griot - Revista de Filosofia, Amargosa, Bahia - Brasil, v.1, n.1, julho / 2010.

NASCIMENTO, L.B.F; ARAÚJO, M.V.P. Práticas de ensino e aprendizagem aplicadas na disciplina de Administração de Materiais do curso de Administração da UFPB. XIII SIMPEP Bauru, SP, Brasil, 6 a 8 de Novembro de 2006.

OCHOA, P; OCHOA, M. A.P. (2010). El saber y las TIC: ¿ Brecha digital o brecha institucional? In: Revista Iberoamericana de Educação, $\mathrm{n}^{\circ}$. 45, Madrid, OEI. Disponível em: <http:// www.rieoei.org/rie53a06.pdf>. Acesso em: mar. 2010.

ROSINI, A. M.; PALMISANO, A. Administração de informação e gestão do conhecimento. São Paulo: Piioneira Thomson Learning, 2003

RUBINSZTEJN G., PALACIOS M (2010). El efecto del tiempo en la percepción de la calidad del servicio educativo. Revista Iberoamericana de Educação, $\mathrm{n}^{\circ} .54$, Madrid, OEI. Disponível em: $<$ http://www.rieoei.org/rie54a09.htm>. Acesso em: mar. 2010.

SCHMITT, C. A. Sistemas integrados de gestão empresarial: uma contribuição no estudo do comportamento organizacional e dos usuários na implantação de sistemas ERP. Tese (Doutorado), Universidade Federal de Santa Catarina, Florianópolis, 2004, 283 p.

SINFO - A Superintendência de Informática da Universidade Federal do Rio Grande do Norte. Projetos. Disponível em: <http://www.info.ufrn.br>. Acesso em 20 jan. 2011.

TACHIZAWA, T.; ANDRADE, R. O. B. de. Tecnologia da informação aplicada às instituições de ensino e às universidades corporativas. $2^{\mathrm{a}}$ ed., São Paulo: Atlas, 2003, 248 p.

VASCONCELOS, M.C.L. Gestão do Conhecimento. Jornal Estado de Minas, Seção Opinião, pp. 09- 29, 12 abr. 2006.

VILCHES, A.; PERÉZ, D.G. (2010). El programa pisa: un instrumento para la mejora del proceso de enseñanza-aprendizaje In: Revista Iberoamericana de Educação, n ${ }^{\circ} .53$, pp. 121 - 154, Madrid, OEI. Disponível em: <http://www.rieoei.org/rie53a06.pdf $>$. Acesso em: mar. 2010. 